\section{and Unraveling the mysteries of reproduction}

\author{
J Fehrer
}

Heredity (2010) 104, 421-422; doi:10.1038/hdy 2010.12; published online 10 March 2010

$\mathrm{U}$ nderstanding the influence of reproductive pathways on population structures has intrigued evolutionary biologists and population geneticists for a long time. Much insight has been gained by mathematical modeling, as well as from empirical case studies of natural populations and from experimental crosses. Among the many factors shaping populations, partially asexual reproduction has received comparatively little attention so far. In an individual-based simulation study in this issue, Navascués et al. (2010) investigated, for the first time, the effect of partial asexual reproduction on the fitness of self-incompatible populations.

Interestingly, most effects were observed only at very high rates $(\geqslant 90 \%)$ of asexuality, and they were strongest in small populations, that is, in which the drift is strongest. In this case, sexual reproduction of self-incompatible plants could even be completely prevented if the number of alleles at the S-locus was reduced to two. Such extreme cases should actually favor the breakdown of self-incompatibility (SI). However, instead of becoming self-compatible, the plants may alternatively go on to reproduce asexually. This could imply that in such cases the number of S-alleles need not be very relevant: it is not necessary that SI disappears, but rather that sexual reproduction may cease, at least temporarily.

There is also empirical evidence that the breakdown of SI may have far more complex causes than merely the number of S-alleles, as exemplified by plants that do not behave as they should according to this theory: In the highly successful colonizer Senecio squalidus, the SI system has been maintained despite a very low number of S-alleles (Brennan et al., 2003). Furthermore, other genetic and non-genetic (for example, environmental) factors have been shown to result in a 'leakiness' of SI systems (Reinartz and Les, 1994, and references therein), and even the developmental stage (age of flowers) can make SI a plastic trait (Mena-Alí et al.,
2009). A further phenomenon (applying to sporophytic SI systems) that indicates that there must be more to the story is the 'mentor effect'; that is, the induced selfing of otherwise self-incompatible individuals under the influence of pollen from other species (for example, Mráz, 2003; Hörandl and Temsch, 2009). This also highlights that physiological and regulatory processes, which are probably not entirely genotype-dependent, might be involved. Given the diversity and versatility of breeding systems and the fact that all of them are 'successful', in the sense that we can observe them, it is also questionable whether the same mechanisms apply to all plant groups.

Partial asexual reproduction not only occurs in plants, but is also widespread in animals and fungi, and, usually, asexual lineages are found to be young derivatives emerging from sexual ancestors. For theoretical reasons, the maintenance of sex ('paradox of sex') is as puzzling as its very opposite, namely the existence of ancient asexual lineages ('scandals'), despite recent advances (for example, Keightley and Otto, 2006). Considering the effects of a large degree of asexuality predicted by Navascués et al. (2010), it is striking that the most drastic effects on nearly all population genetic parameters tested occurred between almost complete (99.9\%) and complete $(100 \%)$ asexuality. This might actually mean that a very small degree of sexual reproduction (or recombination) has tremendous effects. Similar results have been obtained in theoretical (Bengtsson, 2003) as well as empirical studies (D'Souza and Michiels, 2008). The degree of sexuality required to transform entire systems is certainly a topic worth pursuing further.

Models naturally represent simplifications of biological systems. Although much can be learned from such simulations, their biological relevance for understanding real systems needs to be assessed. However, many species have complex lifecycles and, often, too little is known to provide a sound basis for the selection of meaningful population genetics parameters. What is needed are more empirical studies to provide realistic parameter estimates for many different species. These help us to estimate how key parameters vary between different groups of organisms and whether, or to what degree, generalizations are possible. The joint efforts of researchers with different specializations should directly integrate mathematical modeling with empirical case studies of population structure. Ideally, such work should be accompanied by investigations of breeding systems, experimental crosses, and an analysis of the molecular genetic and physiological basis of the relevant traits.

The majority of population genetic studies concentrate on diploids. However, most plants are polyploid, and polyploidy itself (especially in the case of allopolyploid origin) not only has a large range of genomic, cytological, epigenetic and other effects, but also heavily influences population structure. Some clonal plants exhibit very wide variation in the extent of sexual reproduction (Eckert, 2001). For example, in the facultatively apomictic and usually self-incompatible Hieracium subgen. Pilosella, mixed ploidy populations with an enormous versatility of reproductive modes occur regularly. Progeny can be produced sexually via reduced or unreduced male or female gametes, by true apomixis (production of seeds corresponding to the maternal genotype), by vegetative propagation, by haploid parthenogenesis (parthenogenetic development of a reduced egg cell), or by occasional selfing (Krahulcová et al., 2009, and references therein) -in the 'worst case' all by one and the same plant at the same time. Understanding such systems represents a challenging long-term goal for population genetic simulations. Meanwhile, simpler systems starting to deal with polyploids and making a distinction between partially asexual reproduction by vegetative or apomictic propagation (which differ substantially in their dispersal abilities) could be steps in the right direction.

\section{Conflict of interest}

The author declares no conflict of interest.

\section{Acknowledgements}

The Academy of Sciences of the Czech Republic provided financial support (AV0Z60050516). 
$J$ Fehrer is at the Institute of Botany, Academy of Sciences of the Czech Republic, Zámek 1, Priohonice 25243, Czech Republic.

e-mail:fehrer@ibot.cas.cz

Bengtsson BO (2003). Genetic variation in organisms with sexual and asexual reproduction. J Evol Biol 16: 189-199.

Brennan AC, Harris SA, Hiscock SJ (2003). Population genetics of sporophytic self-incompatibility in Senecio squalidus L. (Asteraceae) II: a spatial autocorrelation approach to determining mating behaviour in the presence of low $\mathrm{S}$ allele diversity. Heredity 91: 502-509.

Eckert CG (2001). The loss of sex in clonal plants. Evol Ecol 15: 501-520.

D'Souza TG, Michiels NK (2008). Correlations between sex rate estimates and fitness across predominantly parthenogenetic flatworm populations. J Evol Biol 21: 276-286.

Hörandl E, Temsch EM (2009). Introgression of apomixis into sexual species is inhibited by mentor effects and ploidy barriers in the
Ranunculus auricomus complex. Ann Bot 104: 81-89.

Keightley PD, Otto SP (2006). Interference among deleterious mutations favours sex and recombination in finite populations. Nature 443: 89-92.

Krahulcová A, Rotreklová O, Krahulec F, Rosenbaumová R, Plačková I (2009). Enriching ploidy level diversity: the role of apomictic and sexual biotypes of Hieracium subgen. Pilosella (Asteraceae) that coexist in polyploid populations. Folia Geobot 44: 281-306.

Mena-Alí JI, Keser LH, Stephenson AG (2009). The effect of sheltered load on reproduction in Solanum carolinense, a species with variable self-incompatibility. Sex Plant Reprod 22: 63-71.

Mráz P (2003). Mentor effects in the genus Hieracium s.str. (Compositae, Lactuceae). Folia Geobot 38: 345-350.

Navascués M, Stoeckel S, Mariette S (2010). Genetic diversity and fitness in small populations of partially asexual, self-incompatible plants. Heredity 104: 482-492.
Reinartz JA, Les DH (1994). Bottleneck-induced dissolution of self-incompatibility and breeding system consequences in Aster furcatus (Asteraceae). Am J Bot 81: 446-455.

\section{Editor's suggested reading}

Hörandl E (2009). A combinational theory for maintenance of sex. Heredity 103: 445-457.

Barbará T, Lexer C, Martinelli G, Mayo S, Fay MF Heuertz M (2008). Within-population spatial genetic structure in four naturally fragmented species of a neotropical inselberg radiation, Alcantarea imperialis, A. geniculata, A. glaziouana and A. regina (Bromeliaceae). Heredity 101: 285-296.

Vieira J, Santos RAM, M Ferreira S, Vieira CP (2008). Inferences on the number and frequency of S-pollen gene (SFB) specificities in the polyploid Prunus spinosa. Heredity 101: 351-358. 